\title{
The new face of ovarian cancer modeling: better prospects for detection and treatment
}

\author{
Alison M. Karst and Ronny Drapkin*
}

\begin{abstract}
Addresses: ${ }^{1}$ Department of Medical Oncology, Center for Molecular Oncologic Pathology, Dana-Farber Cancer Institute, 450 Brookline Avenue, Boston, MA 02215, USA; ${ }^{2}$ Department of Pathology, Brigham and Women's Hospital, 75 Francis Street, Boston MA 02115, USA; ${ }^{3}$ Harvard Medical School, 25 Shattuck Street, Boston, MA 02115, USA

*Corresponding author: Ronny Drapkin (ronny_drapkin@dfci.harvard.edu)

FI000 Medicine Reports 20II, 3:22 (doi:10.3410/M3-22)

This is an open-access article distributed under the terms of the Creative Commons Attribution-Non Commercial License (http://creativecommons.org/licenses/by-nc/3.0/legalcode), which permits unrestricted use, distribution, and reproduction in any medium, provided the original work is properly cited. You may not use this work for commercial purposes.

The electronic version of this article is the complete one and can be found at: http://f1000.com/reports/m/3/22

Abstract

Ovarian cancer has a disproportionately high mortality rate because patients typically present with latestage metastatic disease. The vast majority of these deaths are from high-grade serous carcinoma. Recent studies indicate that many of these tumors arise from the fallopian tube and subsequently metastasize to the ovary. This may explain why such tumors have not been detected at early stage as detection efforts have been focused purely on the ovary. In keeping with this leap in understanding other advances such as the development of ex-vivo models and immortalization of human fallopian tube epithelial cells, and the use of integrated genomic analyses to identify hundreds of novel candidate oncogenes and tumor suppressors potentially involved in tumorigenesis now engender hope that we can begin to truly define the differences in pathogenesis between fallopian tube and ovarian-derived tumors. In doing so, we can hopefully improve early detection, treatment, and outcome.
\end{abstract}

\section{The origins of high-grade serous ovarian carcinoma}

Ovarian cancer is currently the fifth leading cause of cancer mortality among American women, responsible for 13,850 deaths in 2010 . Its mortality rate is disproportionately high because it typically goes undetected until patients present with late-stage metastatic disease. Worse still, current screening tools are largely ineffective, making early detection difficult. Currently the standard care for ovarian cancer patients is cytoreductive surgery followed by platinum-taxane chemotherapy, and although most tumors respond to drug treatment, chemoresistant disease typically recurs, resulting in a 5-year survival rate of $~ 30 \%$ for women diagnosed with advanced stage disease. The vast majority of deaths are from high-grade serous ovarian carcinoma.

One of the greatest obstacles in detecting early-stage highgrade serous ovarian carcinoma is our poor understanding of its pathogenesis, and this includes uncertainty surrounding its site of origin. Traditionally, the ovary was thought to be the primary site of high-grade serous ovarian carcinoma tumorigenesis and therefore imagingbased screening techniques have focused on detecting changes in ovary size and shape. However, recent studies indicate that many of the high-grade serous tumors in the pelvis actually arise from fallopian tube fimbria (or possibly the fallopian tube-peritoneal junction) and subsequently metastasize to the ovary [1-7] (figure 1). Figure 2 illustrates the sequence of morphological events described in the fallopian tube that culminate in the development of serous carcinoma (Figure 2). The fact that we may have been looking for early lesions in the wrong anatomical location may explain why so few highgrade serous ovarian carcinoma tumors have been detected at early stage. Herein we will use the term high-grade pelvic serous carcinoma (HGPSC) to account for their possible extra-ovarian origin. The rarity of finding early high-grade serous tumors has also made it difficult to study pre-malignant and early-stage lesions that may shed light on the pathogenesis of this disease. A better understanding of the cell of origin should help 
Figure I. Emergence of serous carcinoma from the fallopian tube and spread to adjacent ovarian surface

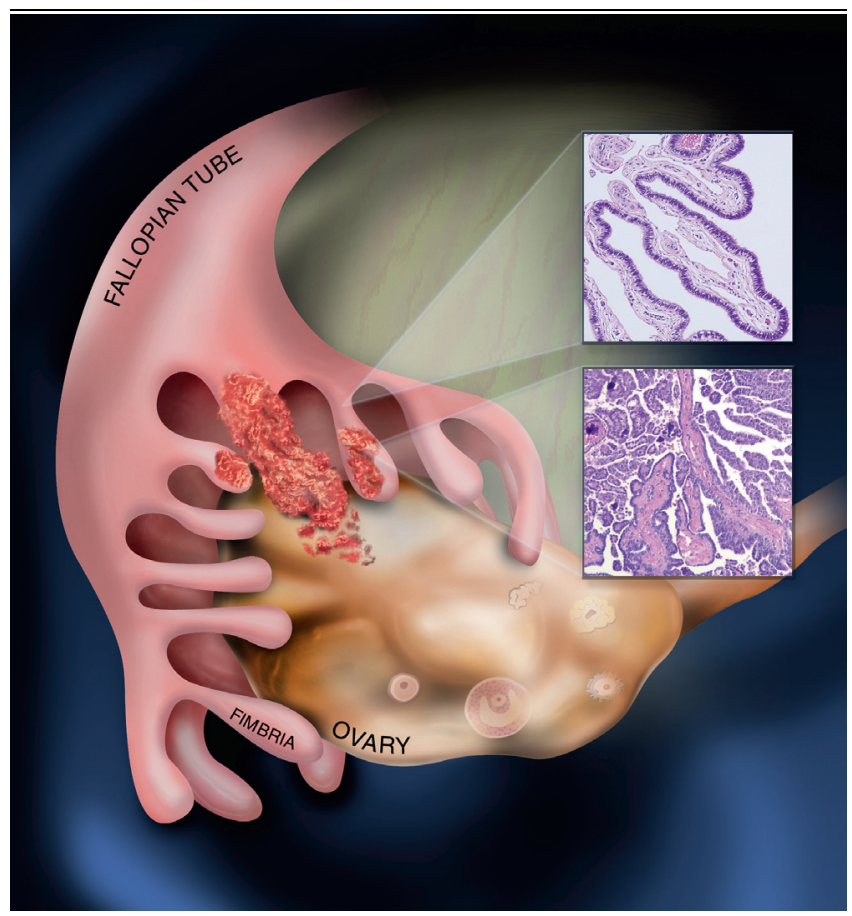

Emerging data suggest that many high-grade serous carcinomas (bottom inset) emerge from secretory cells (top inset) in the fimbriated end of the fallopian tube and spread to the ovarian surface epithelium where the bulk of the tumor resides.

to identify mechanisms of tumorigenesis, predict tumor biology and behavior, and develop strategies for rational intervention.

\section{Elucidating the pathogenesis of high-grade serous carcinoma}

Although the genetics of early-stage HGPSC remain illdefined, the genetics of late-stage tumors have recently been characterized in detail by large-scale integrated genomic analyses conducted by the Cancer Genome Atlas Research Network (CGARN), the Australian Ovarian Cancer Study (AOCS), and the Ovarian Cancer Association Consortium (OCAC) [8-14]. These analyses, as well as earlier smaller scale studies, reveal that high-grade serous tumors are genetically distinct and generally not derived from lower-grade ovarian carcinomas [15-17]. Low-grade ovarian tumors, including low-grade serous, mucinous, and endometrioid carcinomas, are characterized by a spectrum of mutations affecting growth and differentiation pathways. In stark contrast, HGPSCs typically harbor TP53 mutations and deficiencies in homologous recombination repair, resulting in severe genomic instability, evidenced by extensive DNA copy number changes [8, 18-21]. These revelations, however, have generated more questions than answers. For example, does the unique genetic profile of HGPSC result from the tumor cell originating outside the ovary? Or, can HGPSC arise from epithelial cells of either the ovary or fallopian tube? Among the many chromosomal aberrations observed in HGPSC, which genomic alterations initiate and/or drive tumor progression? And perhaps most importantly, which genetic aberrations are required for high-grade serous tumor maintenance and therefore represent targets for therapeutic intervention? To answer these questions it is essential that we develop highfidelity experimental models with which to study the transformation of fallopian tube epithelial cells. In order for these models to be useful, however, it is important that they recapitulate normal biology and can produce tumors that exemplify not only the phenotype of human HGPSC but also its underlying genomic complexity. Recently, significant progress has been made in the development of fallopian tube epithelium-based HGPSC models. Herein we describe these models and discuss their translational impact.

\section{Fallopian tube epithelium-based experimental models \\ Ex-vivo human model}

The "ex vivo" model is a primary cell culture system in which human epithelial cells are isolated from a fresh sample of normal fallopian tube and used to reconstruct an intact fallopian tube epithelium outside of the body [22]. This sophisticated model is based upon one previously developed to study bronchial airway epithelium [23]. Primary ciliated and secretory cells, the two cell types comprising fallopian tube epithelium, are seeded onto a porous transwell cell culture membrane. Remarkably, when seeded onto this membrane, the cells assemble in an organized fashion, assuming their natural orientation, architecture, polarity, extracellular features, and biological functions, including motile cilia (ciliated cells) and protein secretions (secretory cells) (Figure 3). The ex-vivo model is viable for several weeks and is an ideal system for studying normal fallopian tube epithelium biology [24]. It can also be used to examine cellular responses to environmental stress, mechanical damage, DNA damage, cytokines, or inflammatory elements. For example, this model has been used to show that fallopian tube epithelial secretory cells exhibit delayed DNA damage response kinetics, compared to neighboring ciliated cells, following genotoxic insult [22], suggesting that the former cells may be susceptible to accumulating additional DNA damage that could lead to mutations with each subsequent round of injury. Despite the authenticity of the ex vivo model, it is somewhat limited by its small scale and requires a regular supply of primary fallopian tube tissue from which to isolate cells. 
Figure 2. Morphologic development of high grade serous carcinoma from the fallopian tube

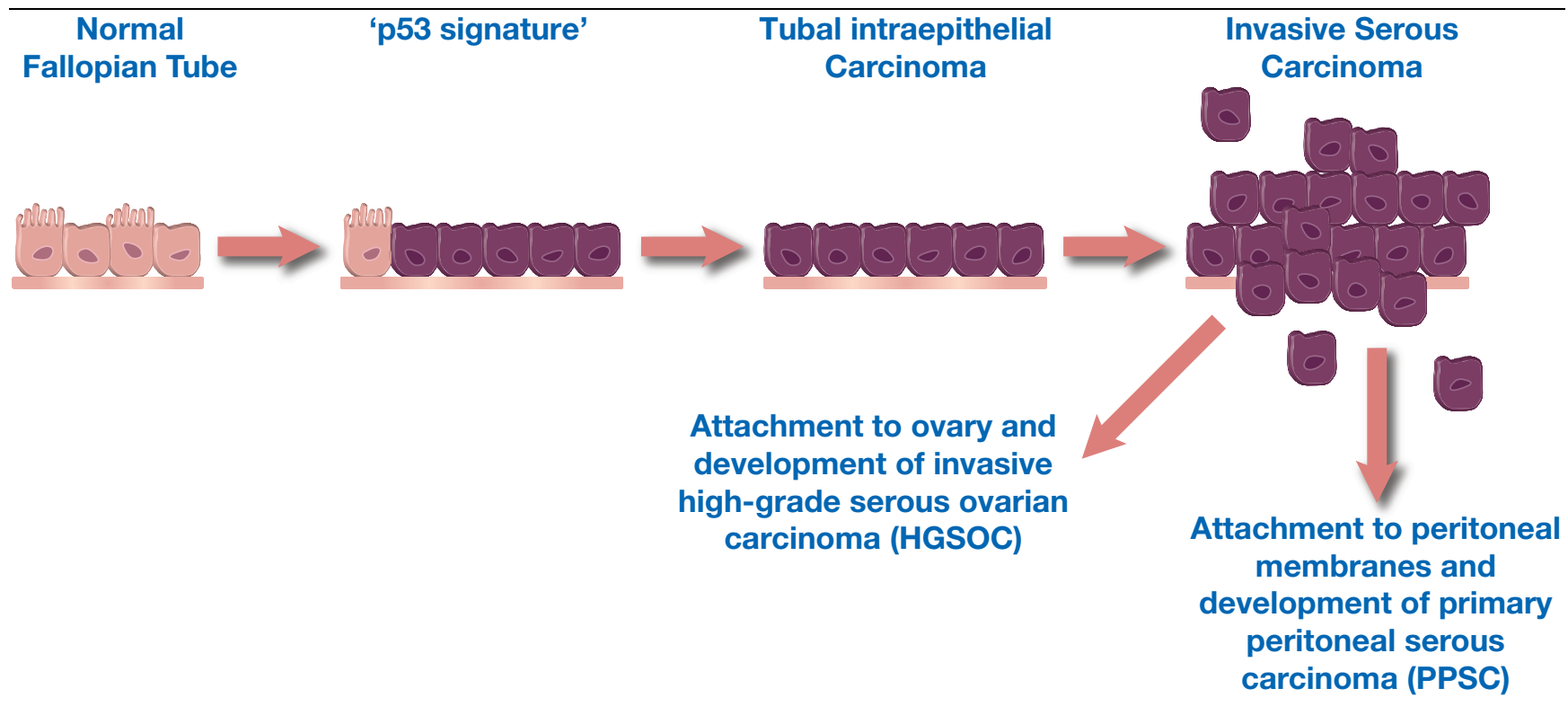

The spectrum of fallopian tube epithelial transformation ranges from normal epithelium with ciliated and secretory cells, through the non-proliferative 'p53 signature' which is characterized by mutations in TP53 and wide-spread DNA damage, to tubal intraepithelial carcinoma (TIC), and eventually, invasive serous carcinoma. Exfoliation of malignant cells is likely to be an early event. This model suggests that most high-grade pelvic serous carcinomas originate from the fallopian tube fimbria, the region of the fallopian tube closest to the ovary.

\section{Immortal human secretory cells}

To study normal fallopian tube epithelium on a larger scale it is necessary to generate primary cell lines. Like most primary human cells, cells of the fallopian tube epithelium inevitably undergo growth arrest and replicative senescence in vitro and therefore must be immortalized for long-term culture. Immortal human ovarian surface epithelial cultures were first established over 15 years ago $[25,26]$ and have been widely used to study biology and cellular transformation. However, immortalization of human fallopian tube epithelial cells has only recently been reported [27] and therefore parallel studies involving these cells have lagged behind. Methods to isolate and culture primary fallopian tube epithelial secretory cells, the presumptive cell of origin of many HGPSC, from fresh samples of normal human fallopian tube have now been published, demonstrating that they can be immortalized by forced expression of telomerase (hTERT) combined with dual targeting of the p53 and pRb pathways.

\section{Transformed human secretory cells}

Integrated genomic analyses of HGPSC [8-14] have identified hundreds of novel candidate oncogenes and tumor suppressors potentially involved in HGPSC tumorigenesis. Due to the extreme genomic complexity of these tumors, however, it is impossible to deduce which genetic alterations are most relevant to the processes of cell transformation, tumor progression, and tumor maintenance, respectively. Recently, largescale siRNA screening has been carried out to identify genes whose amplification or expression is critical for ovarian tumor cell survival and may be promising therapeutic targets [28]. However, analysis of late-stage tumors alone does not provide a comprehensive understanding of HGPSC pathogenesis; we must also identify the genetic events involved in tumor initiation and earlystage progression. One approach to assessing the roles of candidate genes in these processes is to test their ability to induce malignant phenotypes in vitro. It is important that such studies be conducted using non-transformed cells with relatively stable genomes, such as immortal fallopian tube epithelial secretory cells. This approach allows one to systematically evaluate the effects of individual genetic alterations on a normal genetic background and to engineer malignant cells of various genotypes from the same patient sample. Once transformed cell cultures are established in vitro, they are xenografted into mice to assess their tumorigenicity. Using this system, our group recently demonstrated that immortal fallopian tube epithelial secretory cells harboring hTERT, p53, pRb, and PP2A dysfunctions are transformed by additional expression of c-Myc or oncogenic Ras [27]. Upon injection into immunocompromised mice, both c-Myc- and Rastransformed fallopian tube cells gave rise to disseminated tumors that metastasized throughout the peritoneal cavity, 
Figure 3. Ex vivo fallopian tube culture system

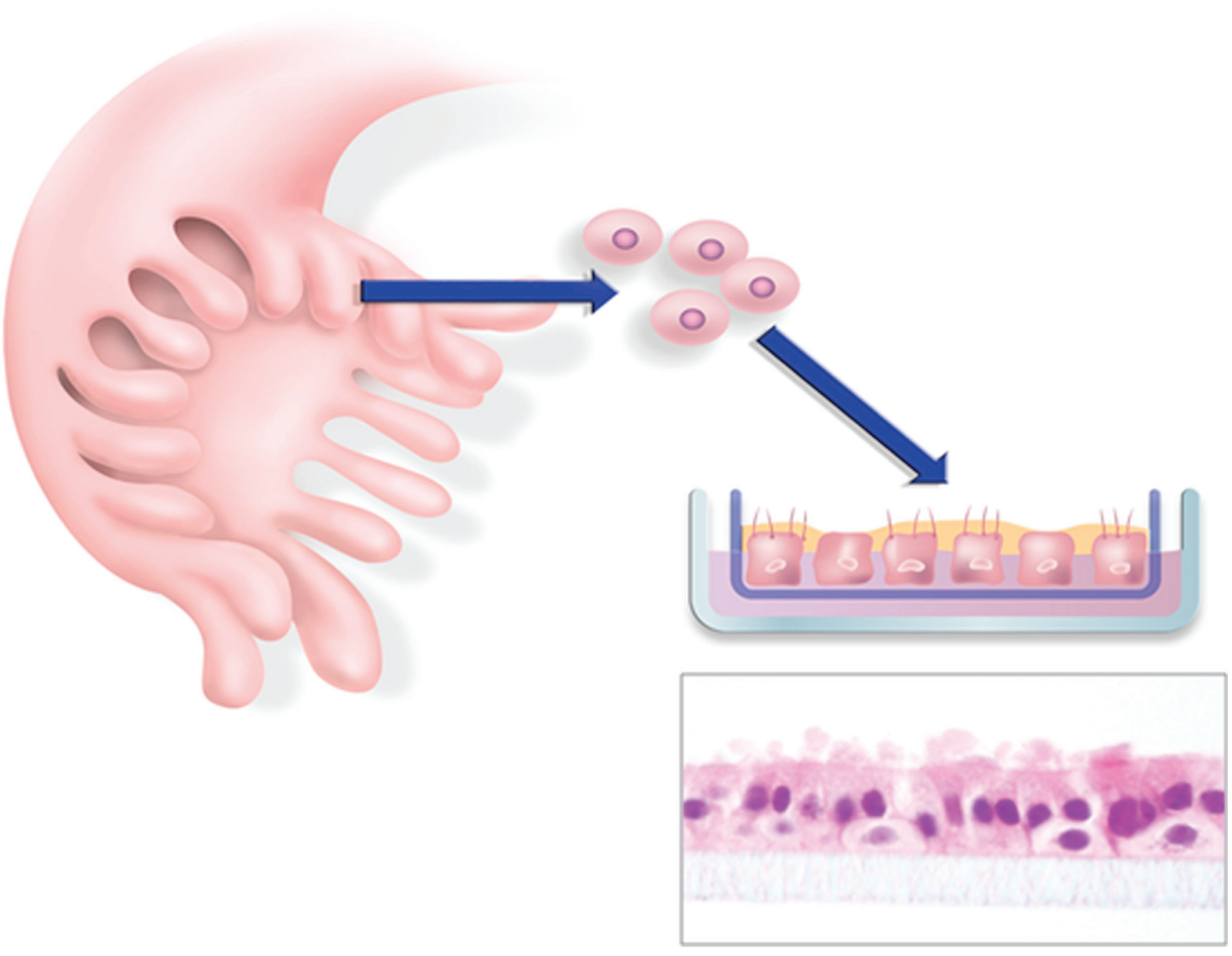

Fallopian tube epithelium is enzymatically dissociated from surgical samples and seeded onto porous transwell filters. The epithelial cells expand to form an epithelial monolayer that faithfully recapitulates the in vivo epithelium, as demonstrated by morphological (the bottom panel is a hematoxylin and eosin stain of a filter removed for microscopic analysis), ultrastructural, immunophenotypic, and biological analyses.

similar to the gross presentation of human HGPSC. The tumors were high-grade carcinomas with a Müllerian immunophenotype, evidenced by Pax8, WT-1, and CK-7 immunohistochemistry positivity, and they expressed the high-grade serous ovarian carcinoma serum biomarkers CA-125 and HE-4, validating that they were indeed serous carcinomas. Array comparative genomic hybridization analysis of tumor tissue revealed widespread DNA copy number changes, indicative of genomic instability and highly reminiscent of the pattern seen in human HGPSC [8-14]. Our system represents the first experimental model of human HGPSC and provides a framework for future studies involving human fallopian tube epithelial secretory cell transformation. In the future, this model can be used to generate high-grade serous tumors with clinically relevant genetic alterations, such as TP53 mutations, BRCA1 dysfunction, and Cyclin E overexpression. These alterations represent the few known early events of fallopian tube tumorigenesis, and such a system should allow us to elucidate their roles.

\section{Transgenic mouse models}

Several transgenic mouse models of ovarian cancer have been developed over the past 10 years, all based on transformation of ovarian surface epithelial cells. Although considerable progress has been made, these models have not fully recapitulated the complexity of HGPSC. It is critical that a transgenic mouse model of fallopian tube epithelium-derived cancer be generated to complement existing ovarian surface epithelial-based 
systems. Unlike ovarian surface epithelial cells, which have no true tissue-specific gene promoters, fallopian tube epithelium secretory cells express highly specific lineage-dependent transcription factors such as Pax8, which may direct transgene expression. Among the most important genes to target in future mouse models will be BRCA1 and BRCA2, the germline mutations linked to familial ovarian and breast cancer, and TP53, which is mutated in nearly $100 \%$ of HGPSC cases. Transgenic mouse models of fallopian tube epithelium-derived HGPSC are currently in development.

Data obtained from the experimental systems described above, when combined with analyses of clinical specimens representing the morphologic continuum from normal fallopian tube epithelium to early tubal carcinomas and then to invasive HGPSC, will allow us to define the genetic events leading to fallopian tube epithelium tumorigenesis and thus significantly advance our understanding of HGPSC pathogenesis.

\section{Translational impact}

Insight gained from fallopian tube epithelium-derived models of HGPSC will have a significant translational impact in several key areas including risk reduction, early detection, and treatment.

Epidemiological studies have long indicated that incessant ovulation is a major HGPSC risk factor. The mechanisms by which ovulatory events contribute to tubal epithelial transformation are unknown but it is hypothesized that follicular rupture during ovulation produces an inflammatory microenvironment containing cytokines and free radicals that may inflict genotoxic damage on nearby cells. Both transgenic mouse models and ex vivo fallopian tube epithelium cultures will be instrumental in exploring the contribution of ovulation and its associated inflammatory factors to fallopian tube epithelium tumorigenesis. Transgenic mouse models will also be key in understanding how germline mutations in BRCA1 or BRCA2 genes confer increased risk of HGPSC and determining whether removal of fallopian tubes alone is sufficient to eliminate increased risk, as has been recently proposed [29].

To reduce the HGPSC mortality rate, it is also imperative that effective screening tools be developed. The ability to screen for early lesions in the fallopian tube using imaging techniques presents a major challenge, as these lesions are extremely small and may shed malignant cells into the peritoneal cavity even at a pre-invasive stage. Therefore, serum biomarker testing remains an optimal means of early detection, since biomarker levels correlate with total tumor mass, regardless of where the tumor cells reside.
Clinically used ovarian cancer biomarkers, CA-125 and HE4, are not sensitive enough to detect early-stage HGPSC and novel biomarkers with higher sensitivity must be identified. Both transgenic mice and human xenograft models of fallopian tube epithelium-derived HGPSC will be critical tools for serum biomarker testing.

Lastly, in order to develop more effective therapies, it is imperative that we utilize high-fidelity HGPSC models for pre-clinical testing. Now that we are gaining comprehensive genetic data from molecular analyses of HGPSC, therapeutic discovery efforts can be focused on targeting the genetic defects that uniquely characterize HGPSC, for example drugs that exploit defective DNA repair pathways and p53 dysfunction. Such novel therapies may be tested on both murine and humanderived tumors generated by transgenic mice and human xenograft models of HGPSC, respectively.

\section{Conclusion}

A great deal of progress has been made in recent years towards understanding the pathogenesis of both ovarian and fallopian tube-derived pelvic serous carcinomas. With the recent development of fallopian tube epitheliumbased experimental models, we can now begin to truly define the differences between fallopian tube epithelium and ovarian surface epithelium-derived tumors as they relate to pathogenesis, biomarker expression, and response to treatment. In doing so, we can hopefully improve early detection, treatment, and outcome for women with ovarian cancer, in particular, HGPSC.

\section{Abbreviation}

HGPSC, High grade pelvic serous carcinoma.

\section{Competing interests}

The authors declare that they have no competing interests.

\section{Acknowledgements}

We thank members of the Drapkin laboratory for fruitful discussions. Our research is supported by a Canadian Institutes of Health Research Fellowship, the National Institutes of Health (U01 CA152990), The Mary Kay Foundation, The Sandy Rollman Ovarian Cancer Foundation, The Ovarian Cancer Research Fund, The Susan Smith Center for Women's Cancers at the Dana-Farber Cancer Institute, and The Robert and Debra First Fund.

\section{References}

I. Kurman RJ, Shih le M: The origin and pathogenesis of epithelial ovarian cancer: a proposed unifying theory. $\mathrm{Am}$ J Surg Pathol 2010, 34:433-43.

FI000 factor 10

Evaluated by Katharina Spanel-Borowski 22 Mar 2010 
2. Karst AM, Drapkin R: Ovarian cancer pathogenesis: a model in evolution. J Oncol 2010, 2010:93237I.

3. Levanon K, Crum C, Drapkin R: New insights into the pathogenesis of serous ovarian cancer and its clinical impact. J Clin Oncol 2008, 26:5284-93.

4. Crum CP, Drapkin R, Miron A, Ince TA, Muto M, Kindelberger DW, Lee $Y$ : The distal fallopian tube: a new model for pelvic serous carcinogenesis. Curr Opin Obstet Gynecol 2007, 19:3-9.

5. Dubeau L: The cell of origin of ovarian epithelial tumours. Lancet Oncol 2008, 9:1191-7.

6. Seidman JD, Zhao P, Yemelyanova A: “Primary peritoneal” highgrade serous carcinoma is very likely metastatic from serous tubal intraepithelial carcinoma: assessing the new paradigm of ovarian and pelvic serous carcinogenesis and its implications for screening for ovarian cancer. Gynecol Oncol. 2011, 1 20:470-3.

7. Seidman JD, Yemelyanova A, Zaino RJ, Kurman RJ: The fallopian tube-peritoneal junction: a potential site of carcinogenesis. Int J Gynecol Pathol 20I I, 30:4- I I.

8. Cancer Genome Atlas Research Network: Integrated genomic analyses of ovarian carcinoma. Nature 20II, 474:609-I5.

FI000 factor 10

Evaluated by Ronny Drapkin 20 Oct 201 I , Carlos Telleria 08 Aug 201 I

9. Pearce CL, Near AM, Van Den Berg DJ, Ramus SJ, Gentry-Maharaj A, Menon U, Gayther SA, Anderson AR, Edlund CK, Wu AH, Chen X, Beesley J, Webb PM, Holt SK, Chen C, Doherty JA, Rossing MA, Whittemore AS, McGuire V, DiCioccio RA, Goodman MT, Lurie G, Carney ME, Wilkens LR, Ness RB, Moysich KB, Edwards R, Jennison E, Kjaer SK, Hogdall E, et al.: Validating genetic risk associations for ovarian cancer through the international Ovarian Cancer Association Consortium. Br J Cancer 2009, 100:4I 2-20.

FI000 factor 6

Evaluated by Ronny Drapkin 20 Oct 2011

10. Fasching PA, Gayther S, Pearce L, Schildkraut JM, Goode E, Thiel F, Chenevix-Trench G, Chang-Claude J, Wang-Gohrke S, Ramus S, Pharoah P, Berchuck A; OCAC (Ovarian Cancer Association Consortium): Role of genetic polymorphisms and ovarian cancer susceptibility. Mol Oncol 2009, 3:|7|-8|.

FI000 factor 6

Evaluated by Ronny Drapkin 20 Oct 2011

II. Goode EL, Chenevix-Trench G, Song H, Ramus SJ, Notaridou M, Lawrenson K, Widschwendter M, Vierkant RA, Larson MC, Kjaer SK, Birrer MJ, Berchuck A, Schildkraut J, Tomlinson I, Kiemeney LA, Cook LS, Gronwald J, Garcia-Closas M, Gore ME, Campbell I, Whittemore AS, Sutphen R, Phelan C, Anton-Culver H, Pearce CL, Lambrechts D, Rossing MA, Chang-Claude J, Moysich KB, Goodman MT, et al.: A genome-wide association study identifies susceptibility loci for ovarian cancer at 2q3 I and 8q24. Nat Genet 2010, 42:874-9.

FI000 factor 6

Evaluated by Ronny Drapkin 20 Oct 2011

12. Etemadmoghadam D, deFazio A, Beroukhim R, Mermel C, George J, Getz G, Tothill R, Okamoto A, Raeder MB, Harnett P, Lade S, Akslen LA, Tinker AV, Locandro B, Alsop K, Chiew YE, Traficante N, Fereday S, Johnson D, Fox S, Sellers W, Urashima M, Salvesen HB, Meyerson M, Bowtell D; AOCS Study Group: Integrated genomewide DNA copy number and expression analysis identifies distinct mechanisms of primary chemoresistance in ovarian carcinomas. Clin Cancer Res 2009, 15:1417-27.

FI000 factor 6

Evaluated by Ronny Drapkin 20 Oct 2011

13. Notaridou M, Quaye L, Dafou D, Jones C, Song H, Høgdall E, Kjaer SK, Christensen L, Høgdall C, Blaakaer J, McGuire V, Wu AH, Van Den Berg DJ, Pike MC, Gentry-Maharaj A, Wozniak E, Sher T, Jacobs IJ, Tyrer J, Schildkraut JM, Moorman PG, Iversen ES, Jakubowska A, Medrek K, Lubiński J, Ness RB, Moysich KB, Lurie G, Wilkens LR,
Carney ME, et al:: Common alleles in candidate susceptibility genes associated with risk and development of epithelial ovarian cancer. Int J Cancer 20 I I, I 28:2063-74.

FI000 factor 6

Evaluated by Ronny Drapkin 20 Oct 2011

14. Bolton KL, Tyrer J, Song H, Ramus SJ, Notaridou M, Jones C, Sher T, Gentry-Maharaj A, Wozniak E, Tsai YY, Weidhaas J, Paik D, Van Den Berg DJ, Stram DO, Pearce CL, Wu AH, Brewster W, AntonCulver H, Ziogas A, Narod SA, Levine DA, Kaye SB, Brown R, Paul J, Flanagan J, Sieh W, McGuire V, Whittemore AS, Campbell I, Gore ME, et al.: Common variants at 19 p I3 are associated with susceptibility to ovarian cancer. Nat Genet 2010, 42:880-4.

FI000 factor 6

Evaluated by Ronny Drapkin 20 Oct 2011

15. Vang R, Shih le M, Kurman RJ: Ovarian low-grade and high-grade serous carcinoma: pathogenesis, clinicopathologic and molecular biologic features, and diagnostic problems. Adv Anat Pathol 2009, I6:267-82.

16. Kuo KT, Guan B, Feng Y, Mao TL, Chen X, Jinawath N, Wang $Y$, Kurman RJ, Shih leM, Wang TL: Analysis of DNA copy number alterations in ovarian serous tumors identifies new molecular genetic changes in low-grade and high-grade carcinomas. Cancer Res 2009, 69:4036-42.

17. Shih leM, Chen L, Wang CC, Gu J, Davidson B, Cope L, Kurman RJ, Xuan J, Wang TL: Distinct DNA methylation profiles in ovarian serous neoplasms and their implications in ovarian carcinogenesis. Am J Obstet Gynecol 2010, 203:584 el-22.

18. Ahmed AA, Etemadmoghadam D, Temple J, Lynch AG, Riad M, Sharma R, Stewart C, Fereday S, Caldas C, Defazio A, Bowtell D, Brenton JD: Driver mutations in TP53 are ubiquitous in high grade serous carcinoma of the ovary. J Pathol 2010, 22 I:49-56.

FI000 factor 6

Evaluated by Ronny Drapkin 20 Oct 2011

19. Press JZ, De Luca A, Boyd N, Young S, Troussard A, Ridge Y, Kaurah P, Kalloger SE, Blood KA, Smith M, Spellman PT, Wang Y, Miller DM, Horsman D, Faham M, Gilks CB, Gray J, Huntsman DG: Ovarian carcinomas with genetic and epigenetic BRCAI loss have distinct molecular abnormalities. BMC Cancer 2008, 8:17.

FI000 factor 6

Evaluated by Ronny Drapkin 20 Oct 2011

20. Turner N, Tutt A, Ashworth A: Hallmarks of 'BRCAness' in sporadic cancers. Nat Rev Cancer 2004, 4:8I4-9.

FI000 factor 6

Evaluated by Ronny Drapkin 20 Oct 2011

21. Mukhopadhyay A, Curtin N, Plummer R, Edmondson RJ: PARP inhibitors and epithelial ovarian cancer: an approach to targeted chemotherapy and personalised medicine. BJOG 201 I, I I 8:429-32.

FI000 factor 6

Evaluated by Ronny Drapkin 20 Oct 2011

22. Levanon $\mathrm{K}, \mathrm{Ng} \mathrm{V}$, Piao $\mathrm{HY}$, Zhang $\mathrm{Y}$, Chang $\mathrm{MC}$, Roh $\mathrm{MH}$, Kindelberger DW, Hirsch MS, Crum CP, Marto JA, Drapkin R: Primary ex vivo cultures of human fallopian tube epithelium as a model for serous ovarian carcinogenesis. Oncogene 2010, 29:। I03-13.

23. Vermeer PD, Einwalter LA, Moninger TO, Rokhlina T, Kern JA, Zabner J, Welsh MJ: Segregation of receptor and ligand regulates activation of epithelial growth factor receptor. Nature 2003, 422:322-6.

FI000 factor 19

Evaluated by Evaluated by Ronny Drapkin 20 Oct 201I, Steven Burden I 4 Jul 2003, Sally Temple0I May 2003, Surender Kharbanda 25 Apr 2003, Charles Streuli 22 Apr 2003, Ben Zion Shilo 01 Apr 2003, Michel Labouesse OI Apr 2003 
24. Fotheringham S, Levanon K, Drapkin R: Ex Vivo Culture of Primary Human Fallopian Tube Epithelial Cells. J Vis Exp 201I, 9:pii 2728.

25. Maines-Bandiera SL, Kruk PA, Auersperg N: Simian virus 40transformed human ovarian surface epithelial cells escape normal growth controls but retain morphogenetic responses to extracellular matrix. Am J Obstet Gynecol 1992, 167:729-35.

FI000 factor 6

Evaluated by Ronny Drapkin 20 Oct 2011

26. Tsao SW, Mok SC, Fey EG, Fletcher JA, Wan TS, Chew EC, Muto MG, Knapp RC, Berkowitz RS: Characterization of human ovarian surface epithelial cells immortalized by human papilloma viral oncogenes (HPV-E6E7 ORFs). Exp Cell Res 1995, 21 8:499-507.

FI000 factor 6

Evaluated by Ronny Drapkin 20 Oct 2011
27. Karst AM, Levanon K, Drapkin R: Modeling high-grade serous ovarian carcinogenesis from the fallopian tube. Proc Natl Acad Sci U S A 20II, 108:7547-52.

28. Cheung HW, Cowley GS, Weir BA, Boehm JS, Rusin S, Scott JA, East A, Ali LD, Lizotte PH, Wong TC, Jiang G, Hsiao J, Mermel CH, Getz G, Barretina J, Gopal S, Tamayo P, Gould J, Tsherniak A, Stransky N, Luo B, Ren Y, Drapkin R, Bhatia SN, Mesirov JP, Garraway LA, Meyerson M, Lander ES, Root DE, Hahn WC: Systematic investigation of genetic vulnerabilities across cancer cell lines reveals lineage-specific dependencies in ovarian cancer. Proc Natl Acad Sci U S A 20I I, 108:12372-7.

29. Greene $M H$, Mai PL, Schwartz PE: Does bilateral salpingectomy with ovarian retention warrant consideration as a temporary bridge to risk-reducing bilateral oophorectomy in BRCAI/2 mutation carriers? Am J Obstet Gynecol 20I I, 204:19 el-6.

FI000 factor 6

Evaluated by Ronny Drapkin 20 Oct 2011 